doppelte Demokratiedefizit der GSVP basiert somit auf einem kontrafaktischen Legitimitätsmodell parlamentarischer Kontrolle, das die empirische Realität innerhalb der EU nicht hinreichend reflektiert.

Bedingt durch den komplementären Charakter der nationalen Parlamente und des Europäischen Parlaments ist die europäische Sicherheits- und Verteidigungspolitik von einer Vielzahl unterschiedlicher Kontrollregime gekennzeichnet, deren individuellen Praktiken zu einem effektiven Mehrebenenmodell parlamentarischer Kontrolle der GSVP zusammenzuführen sind. Es bleibt zu hoffen, dass die letztlich im Rahmen des Protokolls über die Rolle nationaler Parlamente gefundenen institutionellen Arrangements zur Förderung der interparlamentarischen Zusammenarbeit die GSVP tatsächlich transparenter und greifbarer für die europäischen Bürger machen und damit positiv zur demokratischen Legitimität beitragen. Solange aber in jedem Mitgliedsstaat der EU die konstitutionellen und demokratischen Entscheidungsabläufe in Bezug auf die GSVP eingehalten werden - sei es die konstitutive Zustimmung oder lediglich die ex post-Konsultation eines Parlaments - solange muss unter Berücksichtigung der unterschiedlichen nationalen Demokratieverständnisse und den damit verbundenen Legitimitätsvorstellungen der Vorwurf eines doppelten Demokratiedefizits zurückgewiesen werden.

\title{
40 Jahre nach dem ersten Misstrauensantrag im Deutschen Bundestag: Probleme einer Minderheitsregierung am Beispiel der ersten Regierung Willy Brandts
}

Jürgen Plöhn

2012 jährte sich zum 40. Mal die Abstimmung über den ersten Misstrauensantrag der Opposition im Deutschen Bundestag und die erste Vertrauensfrage eines Bundeskanzlers. Am 18. Dezember 2013 wird des 100. Geburtstages Willy Brandts zu gedenken sein. Für Politologen rechtfertigen Jahrestage jedoch nur dann die Erinnerung an zurückliegende Ereignisse, wenn diesen eine fortgesetzte politische Relevanz zukommt. Nach angelsächsischer Tradition gilt das besonders für Präzedenzfälle. Die dramatischen parlamentarischen Ereignisse des Jahres 1972, die in diese Kategorie fallen, sind zwar bereits zeitnah und nachfolgend wiederholt dargestellt worden, die Einordnung der Regierung Brandts als Minderheitsregierung verspricht indes zusätzliche Erkenntnisse.

\section{Zur Abgrenzung: Was ist eine "Minderheitsregierung“?}

Von einer Minderheitsregierung unter Willy Brandt ist in der zeitgeschichtlichen und politikwissenschaftlichen Literatur kaum etwas bekannt ${ }^{1}$. Die parlamentarische Lage im Som-

1 Ludger Helms, Politische Opposition, Opladen 2002, S. 52, nennt die Ereignisse von November/ Dezember 1962 (Konrad Adenauer), Oktober/November 1966 (Ludwig Erhard) und September 1982 (Helmut Schmidt) als die drei einzigen bis 2002 in Deutschland auf Bundesebene aufgetretenen Minderheitsregierungen. 
mer des Jahres 1972 wurde damals unter der Bezeichnung „Patt-Situation“ im Deutschen Bundestag diskutiert ${ }^{2}$. Ludger Helms nennt Brandts Regierung des Jahres 1972 in einer Zusammenstellung deutscher Minderheitsregierungen nicht; Peter Schindler äußert sich ungewohnt widersprüchlich und unklar: Im Text seines Datenhandbuchs wird für 1972 eine Minderheitsregierung „Zwischen dem Patt der Regierungskoalitionen [sic!] und der Auflösung des Bundestages" erwähnt. In der einschlägigen Tabelle findet sich hingegen kein Hinweis auf eine Minderheitsregierung unter Willy Brandt, sondern nur die Randbemerkung, die Koalition sei - allerdings erst drei Wochen nach dem Misstrauensvotum - „ohne Mehrheit" gewesen ${ }^{3}$.

Kaare Strøm definiert als Mehrheitsregierung ein verfassungsgemäß amtierendes Kabinett, das sich aus Vertretern politischer Parteien zusammensetzt, die im Parlament nicht weniger als die Hälfte aller Sitze einnehmen. Er weist ausdrücklich darauf hin, dass er damit auch den Fall eines Gleichstandes der Mandate der Kategorie der Mehrheitsregierung zugeordnet hat, was darin begründet sei, dass ein Kabinett unter diesen Umständen nicht abberufen werden könne ${ }^{4}$. Strøm, der skandinavische und italienische Regierungen vor Augen hat, verfehlt damit allerdings eine Besonderheit des deutschen Verfassungsrechts, die durch die sogenannte „konstruktive" Variante des parlamentarischen Misstrauensvotums geprägt ist: Aufgrund des nach Art. 67 GG uno actu mit der Neuwahl eines Kanzlers erfolgenden Vertrauensentzuges kann nur eine politisch geeinte oppositionelle Mehrheit eine Regierung ablösen.

Der zu Strøms Auffassung gegenteilige Ansatz geht davon aus, dass eine voll handlungsfähige parlamentarische Regierung eine Mehrheit in der Volksvertretung benötigt. Eine solche volle Handlungsfähigkeit ist bei einem Mandatsgleichstand nicht gegeben. Danach ist eine Minderheitsregierung eine Regierung, die nicht Mehrheitsregierung ist und damit für parlamentarische Entscheidungen auf die Zustimmung, zumindest die Enthaltung von Fraktionen, die nicht an der Regierung beteiligt sind, angewiesen bleibt.

Zur Überprüfung des Mehrheits- oder Minderheitscharakters einer Regierung hat Kaare Strøm zwei entscheidende Kriterien benannt: zum einen vertrauensbezogene Abstimmungen, zum anderen parlamentarische Schlussabstimmungen über Gesetzesvorlagen der Regierung 5 .

2 Vgl. Rolf Lange / Gerhard Richter, Erste vorzeitige Auflösung des Bundestages. Stationen vom konstruktiven Mißtrauensvotum bis zur Vereidigung der zweiten Regierung Brandt/Scheel, in: ZParl, 4. Jg. (1973), H. 1, S. $38-75$.

3 Vgl. Peter Schindler, Datenhandbuch des Deutschen Bundestages, 3 Bde, Baden-Baden 1999, Bd. 1, S. $1133 \mathrm{f}$.

4 Strom bezeichnet als „majority cabinet (or government)”: „Any cabinet which meets all appropriate constitutional requirements and which is composed of persons acting as representatives of political parties or parliamentary groups which together control no less than one-half of the total number of seats in the national legislature, or that chamber of the legislature to which the cabinet is politically responsible.” Hierzu erläutert Strom: „Note that we have defined the majority so as to include the even split. The rationale behind this decision is as follows: In order to decide tied votes, many parliaments have mechanisms which favor the side that occupies the institutional leadership, and in parliamentary regimes this should be in the hands of the party or parties in office. Even in the absence of such a bias, the worst predictable outcome for the government would be a stalemate. Under no circumstances does an even split suffice to oust the incumbent government. But the crucial property of a minority government is precisely the counter-intuitive nature of its viability, in that the existing make-up of parliament appears sufficient to bring down the government at any time." Kaare Strom, Minority Government and Majority Rule, PhD-thesis, Stanford 1984, S. 8 f. (mit Anmerkung 11).

5 Vgl. ebenda, S. 7. 
Demgegenüber stellt die deutsche Diskussion stark auf die Existenz einer entsprechenden Parlamentskoalition zwischen verschiedenen Fraktionen $\mathrm{ab}^{6}$, wobei die Einheitlichkeit des Abstimmungsverhaltens aller Mandatsträger einer Partei unterstellt wird. Das entspricht zwar der sogenannten „Parteienstaatstheorie“ des früheren Verfassungsrichters Gerhard Leibholz ${ }^{7}$, wird aber der Wirklichkeit in kritischen Situationen gerade nicht gerecht. Folgt man hingegen Strøm hinsichtlich der konstitutiven Bedeutung von vertrauensbezogenen Abstimmungen, ergibt sich eine erheblich veränderte Perspektive.

\section{Mehrheitsverhältnisse und parlamentarische Demokratie im Frühjahr 1972}

Nach 20 Jahren CDU-geführter Bundesregierungen, zuletzt unter Beteiligung der SPD an der ersten „Großen Koalition“, konnte 1969 mit Willy Brandt nach 39 Jahren wieder ein Sozialdemokrat das Amt des deutschen Kanzlers bekleiden. Willy Brandt und Walter Scheel hatten die im Bundestag neuartige, auf Landesebene allerdings schon verschiedentlich erprobte Zusammenarbeit von Sozialdemokraten und Liberalen angestrebt, obgleich der Parteiflügel des früheren FDP-Vorsitzenden Erich Mende der Neuorientierung der Partei ablehnend gegenüberstand ${ }^{8}$. Dementsprechend erhielt Brandt bei seiner Kanzlerwahl von 254 möglichen Stimmen seiner Parlamentskoalition mit 251 nur wenig mehr als die für die Kanzlerwahl erforderliche absolute Mehrheit von 249 Stimmen?

Innenpolitisch verstand sich die neue Regierungskoalition als Anreger für eine breite Palette von Reformen. Außenpolitisch sollte mit der neuen „Ostpolitik“ mehr als 24 Jahre nach dem Ende des Zweiten Weltkrieges und acht Jahre nach dem Bau der Berliner Mauer ein Neuansatz in den Beziehungen zu den realsozialistisch geprägten Staaten Osteuropas unternommen werden ${ }^{10}$. Basieren sollten die inneren Reformen auf der von Bundeswirtschaftsminister Karl Schiller vertretenen keynesianischen Wirtschaftspolitik ${ }^{11}$ sowie einer rationalen „mittelfristigen Finanzplanung"12.

Aus der Regierungserklärung Brandts ging der Wille zu einem inhaltlichen Neuansatz in der Politik deutlich - und damit polarisierend - hervor. Inhaltlich unscharf setzte Brandt

6 So der Ansatz von Peter Schindler, a.a.O. (Fn. 3), Bd. 1, S. $931 \mathrm{ff}$.

7 Vgl. Gerhard Leibholz, Der Strukturwandel der modernen Demokratie, in: ders., Strukturprobleme der modernen Demokratie, Frankfurt am Main 1974, S. 78 - 129.

8 Auf Landesebene hatte es sozialliberale Koalitionen bereits in Hamburg (1957 bis 1966), Bremen (1959 bis 1971), Berlin (1963 bis 1971) und Nordrhein-Westfalen (1956 bis 1958, seit 1966) gegeben. Zu Bundes- und Landesregierungen Peter Schindler, a.a.O. (Fn. 3), Bd. 1, S. 1439 1462.

9 Aus zeitgeschichtlicher Perspektive hierzu und zum folgenden Arnulf Baring, Machtwechsel. Die Ära Brandt-Scheel, Tb.-Ausgabe, München 1984.

10 Regierungserklärung Willy Brandts abgedruckt bei Karl-Rudolf Korte (Hrsg.), „Das Wort hat der Herr Bundeskanzler“. Eine Analyse der Großen Regierungserklärungen von Adenauer bis Schröder, Wiesbaden 2002, S. 353 - 381; Klaus von Beyme (Hrsg.), Die großen Regierungserklärungen der deutschen Bundeskanzler von Adenauer bis Schmidt, München / Wien 1979, S. 251 - 281.

$11 \mathrm{Zu}$ Schiller und dem von ihm vertretenen Keynesianismus: Peter Hampe (Hrsg.), Friedman contra Keynes. Zur Kontroverse über die Konjunktur- und Beschäftigungspolitik, München 1984; Torben Lütjen, Karl Schiller (1911-1994). „Superminister“ Willy Brandts, Bonn 2008.

12 Zur Finanzpolitik: Wolfgang Jäger, Die Innenpolitik der sozial-liberalen Koalition 1969-1974, in: Karl Dietrich Bracher / Wolfgang Jäger / Werner Link, Die Ära Brandt 1969-1974, Stuttgart / Mannheim 1986, S. 15 - 160, S. 28 f. 


\begin{tabular}{|l|c|c|c|c|c|c|}
\hline \multicolumn{7}{|c|}{ Tabelle 1: Verabschiedung von Gesetzen im Deutschen Bundestag (in Prozent) } \\
\hline & 5. WP & 6. WP & 7. WP & 8. WP & 9. WP & 10. WP \\
& $1965-1969$ & $1969-1972$ & $1972-1976$ & $1976-1980$ & $1980-1983$ & $1983-1987$ \\
\hline Einstimmig & 71,1 & 70,2 & 70,5 & 61,9 & 51,1 & 15,6 \\
Weder einstimmig & - & 23,5 & 23,1 & 30,8 & 39,5 & - \\
noch kontrovers & - & 6,3 & 6,4 & 7,3 & 9,4 & - \\
Kontrovers & - & 6,3 &
\end{tabular}

Anmerkung: In der 5. und 10. Wahlperiode konnten die „weder einstimmig noch kontroversen“ und „kontroversen“ Gesetze nicht ermittelt werden.

Quelle: Peter Schindler, a.a.O. (Fn. 3), Bd. 3, S. 3703.

hinzu: „Wir wollen mehr Demokratie wagen." 13 In der Folgezeit sorgten Außen- und Deutschlandpolitik, Wirtschafts- und Finanzpolitik wie auch die Einschätzung der von Extremisten ausgehenden Risiken für heftige parlamentarische Kontroversen. In der öffentlichen Auseinandersetzung prangerte die Opposition die Regierung als Gefahr für die freiheitlich-rechtsstaatliche Demokratie und die "Soziale Marktwirtschaft" an. Im parlamentarischen Gesetzgebungsverfahren stimmte die Opposition den eingebrachten Gesetzesvorlagen hingegen weitgehend $\mathrm{zu}$ (vgl. Tabelle 1$)^{14}$.

Eine markante Wendung nahm die parlamentarische Arbeit im 6. Bundestag dadurch, dass einige SPD- und FDP-Abgeordnete den Kurs der Koalition aus unterschiedlichen Gründen nicht mittragen konnten und ihre Fraktion verließen ${ }^{15}$. Als die CDU am 23. April 1972 bei der Landtagswahl in Baden-Württemberg erstmals die absolute Mehrheit der Stimmen in diesem Land erzielte, entschloss sich die Unionsfraktion im Bundestag zum Handeln.

Zu dieser Zeit hatte sich die Lage für den Oppositionsführer dadurch zugespitzt, dass die abschließende parlamentarische Beratung der Ostverträge unmittelbar bevorstand. Diese Verträge bildeten zwar in der Öffentlichkeit ein großes Streitthema zwischen Rednern der Regierungsmehrheit und der Opposition; der tatsächliche Frontverlauf war jedoch weit differenzierter. So gehörten in den Reihen der CDU neben dem Oppositionsführer Rainer Barzel auch Richard von Weizsäcker, Norbert Blüm, Erik Blumenfeld, Walther Leisler Kiep und Ernst Majonica trotz mehr oder weniger deutlicher Vorbehalte oder Einwände zu den grundsätzlichen Befürwortern einer auf Ausgleich setzenden, zumindest einem modus vivendi dienenden Ostpolitik, einige - nicht alle - der neugewonnenen Fraktionskollegen hingegen ebenso wie der CSU-Vorsitzende Franz Josef Strauß, der Außenpolitiker Werner Marx oder die Vertriebenenpolitiker Herbert Czaja und Walter Becher zu deren erbitterten Gegnern ${ }^{16}$.

13 Willy Brandt bei Karl-Rudolf Korte (Hrsg.), a.a.O. (Fn. 10), S. 353; Klaus von Beyme (Hrsg.), a.a.O. (Fn. 10), S. 252.

14 Zur kooperativen Oppositionsstrategie der CDU/CSU-Fraktion im 6. Deutschen Bundestag Hans-Joachim Veen, Opposition im Bundestag, Bonn 1976.

15 Vgl. Peter Schindler, a.a.O. (Fn. 3), Bd. 1, S. 931 ff.; Martin Müller, Das konstruktive Mißtrauensvotum. Chronik und Anmerkungen zum ersten Anwendungsfall des Art. 67 GG, in: ZParl, 3. Jg. (1972), H. 3, S. $275-291$.

16 Stark differenzierender Einordnungsversuch bei Christian Hacke, Die Ost- und Deutschlandpolitik der CDU/CSU, Köln 1975, S. 75 f., S. 143; Reinhold Roth, Außenpolitische Innovation und politische Herrschaftssicherung, Meisenheim a.G. 1976, S. 210 f.; mit anderer Gruppierung und kritischer Äußerung zu Richard von Weizsäcker Arnulf Baring, a.a.O. (Fn. 9), S. 446; zur Haltung 


\begin{tabular}{|l|c|}
\hline Tabelle 2: Mehrheitsverhältnisse am 27. April 1972 im Deutschen Bundestag \\
\hline Anzahl der voll stimmberechtigten MdBs & 496 \\
\hline Absolute Mehrheit („Kanzlermehrheit“) & 249 \\
\hline Für Rainer Barzel & 247 \\
Enthaltungen & 3 \\
Damit nicht für Willy Brandt & 250 \\
Für Willy Brandt verblieben & 246 \\
\hline Quelle: Eigene Berechnung nach BT-PIPr 6/183 vom 27. April 1972, S. 10714. \\
\hline
\end{tabular}

Ein Sturz der Regierung Brandt vor der Schlussabstimmung konnte der Opposition die Chance bieten, in Nachverhandlungen die Verträge zu verändern und aufgrund erreichter „Verbesserungen“ das eigene parlamentarische Auseinanderfallen zu vermeiden.

Nach subjektiver Überzeugung der Oppositionsführung hatte die CDU/CSU-Fraktion Ende April 1972 über den Kreis der neuen Fraktionskollegen hinaus feste Zusagen einer hinreichenden Anzahl von Abgeordneten aus den Fraktionen der Regierungsmehrheit, um ein konstruktives Misstrauensvotum nach Art. 67 GG riskieren zu können, wie es die SPD zuvor schon zweimal erfolgreich in Nordrhein-Westfalen und einmal unter etwas anderen rechtlichen Rahmenbedingungen erfolglos in Hamburg gegen die CDU eingebracht hatte ${ }^{17}$. Aufgrund der Abstimmung, an der sich die meisten Abgeordneten der Regierungskoalition nicht beteiligten, blieb Brandt bei 247 Stimmen für und zehn gegen den Antrag sowie drei Enthaltungen gemäß geltendem Verfassungsrecht als Bundeskanzler im Amt, stürmisch umjubelt von Abgeordneten der SPD und FDP. Jedoch hatte er seine vormalige parlamentarische Mehrheit verloren (vgl. Tabelle 2). Barzel hatte trotz der später bekannt gewordenen Bestechungsvorgänge seitens des Ministeriums für Staatssicherheit der DDR, das seine sicher geglaubte absolute Mehrheit verhindern konnte, eine Stimme mehr hinter sich, als der amtierende Bundeskanzler noch auf sich zu vereinen vermochte. Brandt wurde folglich gegen die größere Zahl von Bundestagsabgeordneten durch die Verfassung - unappetitlicherweise im Zusammenspiel mit deren erklärten Feinden in Ost-Berlin ${ }^{18}$ - im Amte gehalten.

Richard von Weizsäckers auch dessen Schreiben vom 26. März 1984 an den Verfasser, in dem Weizsäcker auf seine vorangegangene Beteiligung an der Verbreitung der sogenannten Ost-Denkschrift der EKD hinweist; Barzels Rückschau auf seine Haltung: Rainer Barzel, Auf dem Drahtseil, München / Zürich 1978, S. 66 - 69; ders., Ein gewagtes Leben. Erinnerungen, Stuttgart / Leipzig 2001, S. 282 f., S. $287-293$.

$17 \mathrm{Zu}$ Hamburg: Jürgen Plöhn, Untersuchungsausschüsse der Landesparlamente als Instrumente der Politik, Opladen 1991, S. 160 - 163; zu NRW: Brigitte Dierl / Reinhard Dierl / Heinz-Werner Höffken, Der Landtag von Nordrhein-Westfalen, Bochum 1982, S. 834 - 839; Der Präsident des Landtags Nordrhein-Westfalen (Hrsg.), 40 Jahre Landtag Nordrhein-Westfalen 1946-1986, S. 110 - 113, S. 135 - 138 (Faksimile-Abbildung des Misstrauensantrags von 1966 auf S. 138); vgl. auch Wolfgang Jäger, a.a.O. (Fn. 12), S. 71.

18 Bericht des Untersuchungsausschusses zu den Vorgängen: BT-Drs. 7/1803 vom 13. März 1974; Äußerungen Herbert Wehners im WDR-Fernsehen wiedergegeben in: Frankfurter Rundschau vom 17. Januar 1980; zur Einflussnahme des MfS der DDR „Mischa, Macht und Moral“, in: Der Spiegel vom 28. September 1992, S. 143; „Wie der heilige Georg“, in: Der Spiegel vom 3. Mai 1993, S. 50; CSU-Spion enttarnt, in: Der Spiegel vom 27. November 2000, S. 17; CSU-Politiker bestreitet Stasi-Tätigkeit, in: FAZ vom 28. November 2000; Konrad Schuller, Der „Fall“ Wagner, in: FAZ vom 4. Dezember 2000; Andreas Grau, Auf der Suche nach den fehlenden Stimmen 1972. 
Angesichts der geltenden Rechtslage sah er sich nicht genötigt, wegen seines Vertrauensverlustes zurückzutreten, die Regierungskoalition umzubilden oder unverzüglich Bundestagsneuwahlen einzuleiten ${ }^{19}$. Vielmehr strebte er die Verabschiedung der eingebrachten Gesetzesvorlagen an, darunter insbesondere die Vertragsgesetze zu den Ostverträgen.

\section{Die parlamentarische Situation nach der Abstimmung über den Misstrauensantrag}

\subsection{Die Ostverträge}

Für die Opposition bedeutete der ausgebliebene Wechsel der Bundesregierung nicht nur faktisch eine herbe parlamentarische Niederlage, sondern auch das Problem, ihre außenpolitisch heterogene Koalition aus Gegnern und Befürwortern der Ostpolitik zusammenzuhalten. Die Opposition versuchte die Verabschiedung der Ostverträge durch deren Absetzung von der Tagesordnung des Bundestages hinauszuzögern, erzielte jedoch - unter Einbeziehung der Berliner Abgeordneten - einen Gleichstand von 259 zu 259 Stimmen, der als Ablehnung des gestellten Antrags den Regierungsfraktionen zugute kam.

In der daraufhin angesetzten Schlussabstimmung zu den Verträgen ließ sich die ungeachtet des gemeinsamen Entschließungsantrags aller Fraktionen des Bundestages zu den Ostverträgen ${ }^{20}$ fortbestehende Spaltung der CDU/CSU-Fraktion über deren Inhalt nur durch eine weitgehende Stimmenthaltung bei zugestandener Ablehnung durch einen „harten Kern" von einmal zehn, einmal siebzehn Vertragsgegnern überdecken. Die Regierungsfraktionen konnten ihrerseits unter den voll stimmberechtigten Abgeordneten nur die Hälfte der Stimmen des Bundestages für die Verträge aufbieten. Folglich waren sie faktisch auf die in der Enthaltung liegende passive Unterstützung seitens der Opposition angewiesen. Denn im Falle einer geschlossenen Ablehnungsfront wären die Verträge bei Stimmengleichstand gescheitert $^{21}$; ein mit den Unionsstimmen eingelegter Einspruch des Bundesrates hätte nicht überstimmt werden können. Tatsächlich blieben die Gegenstimmen mit 2,0 Prozent zum Moskauer und 3,4 Prozent zum Warschauer Vertrag deutlich unterhalb der Fraktionsstärke. Auch der Bundesrat ließ die Verträge passieren ${ }^{22}$. Danach sind sie gemäß dem realen Abstimmungsverhalten - trotz der vorangegangenen heftigen verbalen Auseinandersetzungen - nach der Einigung auf eine gemeinsame parlamentarische Entschließung zu ihrer Interpretation denjenigen Beschlüssen zuzuordnen, bei denen die Regierungskoalition seitens der Opposition parlamentarisch nicht herausgefordert worden ist (vgl. Tabelle 3).

Zu den Nachwirkungen des gescheiterten Misstrauensvotums Barzel / Brandt, in: HistorischPolitische Mitteilungen, 16. Jg. (2009), S. 1 - 17.

19 Arnulf Baring berichtet über mehrere Termine, zu denen Brandt die Stellung der Vertrauensfrage erwogen habe. Arnulf Baring, a.a.O. (Fn. 9), S. 449 ff. Faktisch hat Brandt den geeigneten Zeitraum ungenutzt verstreichen lassen.

20 Deutscher Bundestag, 6. WP, Umdruck 287 vom 10. Mai 1972.

21 Vgl. Werner Link, Außen- und Deutschlandpolitik in der Ära Brandt 1969-1974, in: Karl Dietrich Bracher / Wolfgang Jäger / Werner Link, a.a.O. (Fn. 12), S. 163 - 282, S. 206 - 213; ders., Die CDU/CSU-Fraktion und die neue Ostpolitik - in den Phasen der Regierungsverantwortung und der Opposition, 1966-1975, in: Hans-Peter Schwarz (Hrsg.), Die Fraktion als Machtfaktor. CDU/ CSU im Deutschen Bundestag 1949 bis heute, Bonn / München 2010, S. 115 - 139, S. $131 \mathrm{f}$.

22 Bundesrat, 381. Sitzung am 19. Mai 1972. 


\subsection{Der Bundeshaushalt}

Zeigt sich der Misstrauensantrag und die Entstehung der Minderheitskanzlerschaft danach in besonderer Weise mit der Ostpolitik verknüpft, ergibt sich für die Auflösung der entstandenen Problematik ein enger Zusammenhang mit finanzpolitischen Entscheidungen.

Schon vor der Abstimmung über den Misstrauensantrag war angesichts der schwindenden Regierungsmehrheit in der Presse über Neuwahlen spekuliert worden ${ }^{23}$. Der Opposition erschien diese Option nach ihrer Abstimmungsniederlage indes inopportun. Vielmehr ging sie in abruptem Strategiewechsel noch am Tage der Abstimmung über den Misstrauensantrag zu einer "Tolerierung“ der Bundesregierung über, indem sie Bereitschaft zu Absprachen zeigte, worauf Willy Brandt am folgenden Tage im Deutschen Bundestag einging:

„Im gestrigen Gespräch, das wir mit den Kollegen Barzel und Stücklen führten, haben Herr Scheel und ich nicht den Eindruck gewonnen, als ob die CDU/CSU ein aktuelles Interesse an dieser Frage [vorgezogene Bundestagswahlen] hätte. Dabei wissen wir aber doch: Im Grunde ermöglicht unsere Verfassung Neuwahlen ohne zusätzlichen Streit und Krampf nur dann, wenn sich alle Fraktionen des Hohen Hauses auf ein gemeinsames Vorgehen verständigen." 24

Die ausgedrückte Rechtsauffassung, die vom Verfassungstext nicht gedeckt war und höchstens verfassungspolitisch auf eine Änderung des Grundgesetzes hat abzielen können, ist für den Fortgang der deutschen Geschichte angesichts der in diesem Punkte festen, auf die geltende Verfassung bezogenen Haltung der Opposition nicht relevant geworden. Prägend aber wurden Brandts Bemerkungen zu den finanziellen Möglichkeiten seiner Regierung. Angesichts der drohenden Niederlage über eine Haushaltsabstimmung formulierte der Kanzler:

„Es stellt sich ... aus meiner Sicht die Frage, ob in einer Situation mit knappen Mehrheiten zur gemeinsamen Verantwortung nicht auch gehört, daß eine Opposition der Versuchung widersteht, nach einem gescheiterten Mißtrauensvotum ihren Versuch an dem empfindlichen Objekt des Haushalts zu wiederholen ... (...) ... Dazu darf ich in Paranthese [sic!] bemerken, daß die Bundesregierung nach den Art. 111 und 112 GG ohnehin haushaltsmäßig genügend Spielraum hat, aus eigener Verantwortung ihre Pflichten zu erfüllen." 25

Die von Brandt befürchtete Niederlage realisierte sich nur Stunden später am 28. April 1972 durch einen ersten Stimmengleichstand ( $247 \mathrm{zu} 247$ bei einer Enthaltung) zu dem für den Bundeskanzler vorgesehenen Etatposten 0426. Daraufhin wurde der Bundeshaushalt für das Jahr 1972 am 7. Juni an den Haushaltsausschuss des Bundestages zurücküberwiesen ${ }^{27}$.

Mit seiner Intervention hatte der Kanzler erstens versucht, zum eigenen Machterhalt die Opposition von der Nutzung ihrer verfassungsmäßigen Rechte abzubringen. Zweitens erklärte er, elementare parlamentarische Kompetenzen umgehen zu können. Pikanterweise konserviert Artikel 111 Abs. 1 GG verfassungsrechtlich das Ergebnis des so genannten Preußischen Verfassungskonflikts um die Heeresreform, den Otto von Bismarck in den 60er

23 Vgl. RolfLange/ Gerhard Richter, a.a.O. (Fn. 2), S. 39.

24 Willy Brandt, BT-PIPr 6/184 vom 28. April 1972, S. 10759.

25 Willy Brandt, BT-PIPr 6/184 vom 28. April 1972, S. 10758 f.

26 BT PIPr 6/184 vom 28. April 1972, S. 10787.

27 BT PIPr 6/188 vom 7. Juni 1972, S. 11064. 
Jahren des 19. Jahrhunderts an der Seite König Wilhelms I. gegen das Parlament ausgefochten hatte ${ }^{28}$. In scharfem Gegensatz zur US-amerikanischen Verfassungspraxis ist nach dieser Grundgesetznorm die Regierung zur vorläufigen Haushaltsführung berechtigt, um rechtlichen Verpflichtungen und begonnenen Projekten nachzukommen ${ }^{29}$. Der Einigungszwang ist dadurch in Deutschland drastisch geringer als in den USA, wo der Präsident ohne zuvor erteilte Bewilligung durch den Kongress der Staatskasse keinen Cent entnehmen darf ${ }^{30}$.

Als Ausnahmenorm ist die Vorschrift des Art. 111 Abs. 1 GG nach den gängigen Regeln der Verfassungsinterpretation eng auszulegen. Brandt hat sie jedoch gegen das Budgetrecht des Parlaments ins Spiel gebracht und faktisch zur Grundlage seiner Haushaltsführung gemacht. Zugleich ist diese Ausnahmenorm durch die Erwähnung der weiteren Ausnahmeregelung des Art. $112 \mathrm{GG}^{31}$ von Brandt noch deutlich erweitert worden.

Während zuvor geradezu regelmäßig der Haushaltsplan mehrere Monate verspätet in Kraft getreten ist, verzögerte sich die Verabschiedung des Bundeshaushalts für 1972 letztlich um ein ganzes Jahr. Denn erst das nächste Parlament verabschiedete den Etat - und zwar am 20. Dezember 1972, so dass er am 29. Dezember im Bundesgesetzblatt verkündet wur$\mathrm{de}^{32}$. Vor dem Hintergrund solch obrigkeitsstaatlicher Praktiken fragte Winfried Steffani

28 Vgl. Jürgen Schlumbohm (Hrsg.), Der Verfassungskonflikt in Preußen 1862-1866 (Historische Texte/ Neuzeit), Göttingen 1970; Hans Boldt, Deutsche Verfassungsgeschichte Bd. 2, München 1993, S. 108 - 119; Ernst-Rudolf Huber, Deutsche Verfassungsgeschichte seit 1789, Bd. III: Bismarck und das Reich, Stuttgart u.a. 1970, S. 305 - 369, zur „Lückentheorie“, S. 333 - 341. Die Textgeschichte kann nur bis zur preußischen Verfassung von 1920 zurückgeführt werden, da die Vorschrift, inhaltlich auf das monarchische Prinzip gestützt, bis zum Ende des Kaiserreiches keiner rechtlichen Formalisierung bedurfte. Christoph Gröpl, Art. 111, in: Rudolf Dolzer / Klaus Vogel / Karin Graßhoff (Hrsg.), Bonner Kommentar zum Grundgesetz, Bd. 10, Heidelberg 2001 (Stand: Dezember 2011), S. 8, Rdnr. 2; Markus Heintzen, $\$ 120$ Staatshaushalt, in: Josef Isensee / Paul Kirchhof (Hrsg.), Handbuch des Staatsrechts der Bundesrepublik Deutschland, Bd. V, Heidelberg 2007, S. 1175 - 1225, Rdnr. 9-10.

29 Art. 111 [Ausgaben vor Genehmigung des Etats]: (1) Ist bis zum Schluß eines Rechnungsjahres der Haushaltsplan für das folgende Jahr nicht durch Gesetz festgestellt, so ist bis zu seinem Inkrafttreten die Bundesregierung ermächtigt, alle Ausgaben zu leisten, die nötig sind, a) um gesetzlich bestehende Einrichtungen zu erhalten und gesetzlich beschlossene Maßnahmen durchzuführen, b) um die rechtlich begründeten Verpflichtungen des Bundes zu erfüllen, c) um Bauten, Beschaffungen und sonstige Leistungen fortzusetzen oder Beihilfen für diese Zwecke weiter zu gewähren, sofern durch den Haushaltsplan eines Vorjahres bereits Beträge bewilligt worden sind.

30 Artikel I Section 9 Abs. 7 US-Verfassung: „No Money shall be drawn from the Treasury, but in Consequence of Appropriations made by Law; and a regular Statement and Account of the Receipts and Expenditures of all public Money shall be published from time to time." Hierzu Winfried Steffani, Seniorität im amerikanischen Kongreß: Das Beispiel Cannon, in: ders., Parlamentarische und präsidentielle Demokratie, Opladen 1979, S. 346 - 358, vor allem S. 351; Patrick Horst, Haushaltspolitik und Regierungspraxis in den USA und der Bundesrepublik Deutschland, Frankfurt am Main 1995.

31 Art. 112 [Haushaltsüberschreitung]: Überplanmäßige und außerplanmäßige Ausgaben bedürfen der Zustimmung des Bundesministers der Finanzen. Sie darf nur im Falle eines unvorhergesehenen und unabweisbaren Bedürfnisses erteilt werden. Näheres kann durch Bundesgesetz bestimmt werden.

32 Gesetz über die Feststellung des Bundeshaushaltsplans für das Haushaltsjahr 1972 (Haushaltsgesetz 1972) vom 21. Dezember 1972, in: BGBl., Teil I Nr. 142 vom 29. Dezember 1972, S. 2537 - 2541; hierzu Bundesministerium der Finanzen (Hrsg.), Finanzbericht 1973, Bonn 15. März 1973, S. 91. 
bereits in den siebziger Jahren, ob das versprochene Mehr an Demokratie auch für die Opposition gelten solle ${ }^{33}$.

\subsection{Die Bilanz der Gesetzgebung}

Betrachtet man die Gesamtbilanz der gesetzgeberischen Arbeit des Bundestages zwischen Misstrauensvotum und Vertrauensfrage, finden sich lediglich zwei Gesetze (3,2 Prozent aller Schlussabstimmungen dieser Periode), die in ihrer parlamentarischen Schlussabstimmung gegen den Widerspruch der Oppositionsfraktion angenommen worden sind. Nach dem Scheitern des Misstrauensantrages scheint Konsens das Parlament beherrscht zu haben, wobei in drei Fällen sogar oppositionelle Gesetzesvorschläge einvernehmlich beschlossen wurden (vgl. Tabelle 3).

\begin{tabular}{|l|c|c|c|c|c|}
\hline \multirow{2}{*}{ Tabelle 3: Abstimmungsmaterien im Deutschen Bundestag, 27. April bis 22. September 1972} \\
\hline \multirow{2}{*}{ Abstimmungsmaterie } & \multicolumn{3}{|c|}{ Verbindliche Beschlussfassung (Schlussabstimmung) } \\
\cline { 2 - 6 } & \multicolumn{2}{|c|}{ Kontrovers } & \multicolumn{2}{|c|}{ Nicht-kontrovers } \\
\cline { 2 - 6 } & absolut & in Prozent & absolut & in Prozent \\
\hline \multirow{2}{*}{$\begin{array}{l}\text { Gesetze (innerstaatliche Regelungen) } \\
\text { davon i. S. d. Regierungsmehrheit } \\
\text { davon i. S. d. Opposition }\end{array}$} & 2 & & & & \\
\hline Vertragsgesetze (internationale Verträge) & 0 & 0 & 42 & 39 & 88,6 \\
\hline Parlamentarische Entschließungen & 0 & 0 & 5 & 6,8 \\
\hline * Einschließlich zweier Abstimmungen ohne oppositionelle Herausforderung der Regierungsseite im \\
$\begin{array}{l}\text { Parlament (,uncontested“). } \\
\text { Quelle: Eigene Berechnung nach BT-PIPr 6/183-6/199. }\end{array}$
\end{tabular}

Trotz der statistisch hohen Zustimmungsrate seitens der Opposition handelte es sich bei Brandts Regierung jedoch nicht um eine von der CDU/CSU-Fraktion „gestützte“ und damit stabile, parlamentarisch mehrheitsfähige Regierung ${ }^{34}$, denn erstens passten sich die Regierungsfraktionen der Lage an, indem sie zu verabschieden suchten, wofür Übereinstimmung vorhanden war ${ }^{35}$. Zweitens differenziert sich das Bild, wenn man nicht nur die Schlussabstimmungen betrachtet, sondern auch Detailberatungen mit einbezieht, wie sie während der 2. Lesung von Gesetzesvorhaben stattfinden.

33 Vgl. Winfried Steffani, Mehr Demokratie auch für die Opposition?, in: ders., Parlamentarische und präsidentielle Demokratie, Opladen 1979 (Erstveröffentlichung 1977), S. 250 - 262. Mit Bezug auf den budgetlosen Zustand angesichts des Verstoßes gegen das Demokratieprinzip im konkreten Fall kritisch Eberhard Fricke, Über die Pflicht zur Haushaltsgesetzgebung, in: DVBl. 90. Jg. (1975), S. $604-607$.

34 Vgl. Eberhard Schütt-Wetschky, Verhältniswahl und Minderheitsregierungen. Unter besonderer Berücksichtigung Großbritanniens, Dänemarks und der Bundesrepublik Deutschland, in: ZParl, 18. Jg. (1987), H. 1, S. $94-109$, S. 105 f.

35 Darunter: erste Erweiterung der Europäischen Gemeinschaften, BT-PIPr 6/194 vom 21. Juni 1972; dagegen: Zurückziehung der noch nicht entscheidungsreifen Intelsat-Vorlage, BT-PIPr 6/199 vom 22. September 1972, S. 11738. 


\begin{tabular}{|l|l|}
\hline \multicolumn{2}{|c|}{ Tabelle 4: Namentliche Abstimmungen und Auszählungen im Deutschen Bundestag, 27. April } \\
bis 22. September 1972
\end{tabular} \mid \begin{tabular}{l}
\multicolumn{1}{|c|}{ Ausgang / Vorteil } \\
\hline Anzahl
\end{tabular}

\section{Tabelle 5: Abstimmungserfolge im Deutschen Bundestag, 27. April bis 22. September 1972}

\begin{tabular}{|l|c|l|}
\hline Politischer Erfolg & Anzahl & \multicolumn{1}{c|}{ Bemerkungen } \\
\hline Regierungsfraktionen & $\begin{array}{r}6 \\
+1\end{array}$ & $\begin{array}{l}2 \times \text { ohne oppositionelle Herausforderung, } \\
1 \times \text { Erfolg als Minderheit (laut GG) }\end{array}$ \\
\hline Oppositionsfraktion & 8 & $\begin{array}{l}1 \times \text { ohne regierungsseitige Herausforderung, } \\
1 \times \text { ohne Erfolg trotz relativer Mehrheit }\end{array}$ \\
\hline \multirow{2}{*}{ Konsens } & 3 & $\begin{array}{l}1 \times \text { Zustimmung beider Seiten erforderlich (laut GG), } \\
2 \times \text { politisch von Opposition erzwungen }\end{array}$ \\
\hline \multicolumn{2}{|l}{ Quelle: Eigene Berechnung nach PIPr 6/183-6/199. } \\
\hline
\end{tabular}

Wertet man alle 17 Abstimmungen aus, bei denen die Stimmen gezählt oder namentlich registriert worden sind, ist Konsens selten zu finden. Auffällig ist vielmehr die breite Auffächerung vorkommender Mehrheitsverhältnisse (vgl. Tabelle 4). In der Summe haben die Regierungsfraktionen sechsmal Mehrheiten oder einen für sie günstigen Gleichstand erzielt. Die Niederlage bei der Abstimmung zum Misstrauensantrag hat sich nicht gegen sie ausgewirkt. Für die Opposition ist die hierbei erreichte relative Mehrheit hingegen eine politische Niederlage gewesen. Nimmt man die drei einvernehmlichen Abstimmungen hinzu, für die in zwei Fällen die Anregungen von der Opposition, einmal von der Regierungsseite ausgegangen sind, kommt man für Regierungsfraktionen und Opposition insgesamt zu einer Bilanz von acht zu neun politischen Erfolgen (vgl. Tabelle 5). 
3.4. Politische Aktivitäten in der parlamentarischen Sommerpause und das Ende der Wahlperiode

Obgleich die gegen Brandt optierenden FDP-Abgeordneten Gerhard Kienbaum und Knut von Kühlmann-Stumm angesichts der Perspektivlosigkeit ihrer Position im Frühjahr 1972 nach der Misstrauensabstimmung ihre Mandate niedergelegt hatten ${ }^{36}$, blieb die Regierung für parlamentarische Erfolge bis zum Ende der Wahlperiode weitgehend auf die Unterstützung der Opposition angewiesen. Nach kurzfristiger Besserung der Lage des Kanzlers aufgrund zweier loyaler Nachrücker in der FDP verschlechterte sich seine Situation durch Konflikte um Bundeswirtschafts- und Finanzminister Karl Schiller erneut. Denn nach seinem Rücktritt vom Amte nahm Schiller auch sein Bundestagsmandat nicht mehr wahr, jedoch ohne es niederzulegen. Die Führungsschwäche des Kanzlers in seinem Kabinett wirkte sich damit nach der parlamentarischen Sommerpause in den letzten drei Plenarsitzungen des Bundestages auch auf dessen parlamentarische Mehrheit aus ${ }^{37}$.

Zugleich ließ die Schwäche der Regierung der Opposition Raum für eine eigene „Nebenaußenpolitik“, für die Gerhard Schröder (CDU) als ehemaliger Außenminister und Vorsitzender des Auswärtigen Ausschusses des Deutschen Bundestages über die geeignete Statur und formale Position verfügte. Seine Reise in die Volksrepublik China mit ranghohen Gesprächspartnern störte im Juli 1972 die Aussöhnungslinie der Regierung gegenüber der Sowjetunion. Von der Opposition unter Zugzwang gesetzt, besuchte Bundesaußenminister Walter Scheel im Oktober das Land und vereinbarte in Anknüpfung an Schröders Sondierungen nach formellen Verhandlungen die Aufnahme diplomatischer Beziehungen ${ }^{38}$.

Der Weg zur Neuwahl des Bundestages, in der die sozialliberale Koalition unter Brandt einen glänzenden Sieg erringen konnte, führte über die so genannte Vertrauensfrage des Bundeskanzlers und die auf dessen Antrag hin erfolgende Auflösung des Parlaments durch den Bundespräsidenten, wie sie dann zu einer gelegentlichen, 1982 und 2005 genutzten Verfassungspraxis geworden ist ${ }^{39}$.

Effektiv hat Willy Brandt bei der Abstimmung über die parlamentarische Vertrauensfrage am 22. September 1972 das politische Vertrauen einer Mehrheit der Bundestagsabgeordneten nicht mehr gehabt. Die Nichtbeteiligung der Regierungsmitglieder an der Abstimmung hat diesen Umstand vor der Öffentlichkeit zwar etwas verschleiern können, das Ergebnis von 233 (+12) Ja-Stimmen zu 248 (+10) Nein-Stimmen bei - offenbar irrtümlicher - Enthaltung des Bundesministers Walter Arendts bedeutet indes, dass auch bei Beteiligung aller

36 Angaben zur Mandatsniederlegung bei Peter Schindler, a.a.O. (Fn. 3), Bd. 3, S. 4042, S. 4051; öffentliche Bekanntgabe durch den Bundestagspräsidenten: BT-PIPr 6/185 vom 4. Mai 1972 und 6/188 vom 7. Juni 1972.

37 Vgl. Torben Lütjen, a.a.O. (Fn. 11), S. 332 - 356; Wolfgang Jäger, a.a.O. (Fn. 12), S. 52.

38 Vgl. Hermann Schreiber, Ein Königskind auf Reisen, in: Der Spiegel vom 24. Juli 1972, S. 22 f.; „Das chinesische Meer ist tief", in: Der Spiegel vom 7. August 1972, S. 19 - 24; Günter Gaus, Ausgerechnet Herr Schröder, in: ebenda, S. 20; Mechthild Leutner (Hrsg.), Bundesrepublik Deutschland und China 1949 bis 1995. Politik - Wirtschaft - Wissenschaft - Kultur. Eine Quellensammlung, Berlin 1995, S. 142, S. 147 - 149.

39 Vgl. Jürgen Plöhn, „Konstruktives Mißtrauensvotum“ und „Vertrauensfrage“ im internationalen Vergleich, in: ders. (Hrsg.), Sofioter Perspektiven auf Deutschland und Europa. Studien zu Wirtschaft, Politik, Geschichte, Medien und Kultur, Münster 2006, S. 127 - 165, S. 152 ff.; Michael Feldkamp, Chronik der Vertrauensfrage des Bundeskanzlers am 1. Juli 2005 und der Auflösung des Deutschen Bundestages am 21. Juli 2005, in: ZParl, 37. Jg. (2006), H. 1, S. 19 - 28. 


\begin{tabular}{|c|c|c|c|c|c|}
\hline \multirow[t]{2}{*}{ Tabelle 6: } & \multicolumn{5}{|c|}{$\begin{array}{l}\text { Entwicklung der Beitragseinnahmen und Rentenausgaben in der gesetzlichen Renten- } \\
\text { versicherung, } 1970 \text { bis } 1975 \text { (in Millionen DM) }\end{array}$} \\
\hline & $\begin{array}{l}\text { Beitrags- } \\
\text { einnahmen }\end{array}$ & $\begin{array}{l}\text { Renten- } \\
\text { ausgaben }\end{array}$ & $\begin{array}{l}\text { Veränderung } \\
\text { der Beiträge }\end{array}$ & $\begin{array}{l}\text { Veränderung } \\
\text { der Renten- } \\
\text { zahlungen }\end{array}$ & $\begin{array}{c}\text { Differenz der } \\
\text { Veränderungen } \\
\text { zum Vorjahr }\end{array}$ \\
\hline \multicolumn{6}{|c|}{ RV Arbeiter } \\
\hline 1970 & 25.740 & 24.414 & - & - & \\
\hline 1971 & 29.112 & 26.339 & +3.372 & +1.925 & +1.447 \\
\hline 1972 & 31.804 & 29.893 & +2.692 & +3.554 & -862 \\
\hline 1973 & 37.178 & 35.058 & +5.374 & +6.165 & -791 \\
\hline 1974 & 40.132 & 40.551 & +2.954 & +5.493 & -2.539 \\
\hline 1975 & 41.049 & 45.680 & +917 & +5.129 & -4.212 \\
\hline \multicolumn{6}{|c|}{ RV Angestellte } \\
\hline 1970 & 16.648 & 13.979 & - & - & \\
\hline 1971 & 19.549 & 15.168 & +2.901 & +1.189 & +1.712 \\
\hline 1972 & 23.143 & 17.328 & +3.594 & +2.160 & +1.430 \\
\hline 1973 & 27.940 & 20.192 & +4.797 & +2.864 & +1.933 \\
\hline 1974 & 31.965 & 23.703 & +4.025 & +3.511 & +514 \\
\hline 1975 & 36.223 & 27.152 & +4.258 & +3.449 & +809 \\
\hline \multicolumn{6}{|c|}{ Knappschaftliche RV } \\
\hline 1970 & 1.230 & 5.115 & - & - & \\
\hline 1971 & 1.353 & 5.386 & +123 & +271 & -148 \\
\hline 1972 & 1.350 & 5.803 & -3 & +417 & -420 \\
\hline 1973 & 1.420 & 6.362 & +70 & +559 & -489 \\
\hline 1974 & 1.607 & 7.020 & +187 & +658 & -471 \\
\hline 1975 & 1.755 & 7.667 & +148 & +647 & -499 \\
\hline
\end{tabular}

14 Kabinettsmitglieder Bundeskanzler Brandt eine - in diesem Falle so akzeptierte - 247-zu248-Niederlage erlitten hätte ${ }^{40}$.

\section{Die finanziellen Konsequenzen der Minderheitsregierung}

Hinsichtlich der zur Wiederherstellung einer voll handlungsfähigen Regierungsmehrheit erforderlichen Neuwahl zeigten sich alle im Bundestag vertretenen Parteien insoweit „staatstragend“, als sie sich für die Bundesrepublik Deutschland 1972 im Kontrast zu den unter dem Nationalsozialismus abgehaltenen Berliner Sommerspielen von 1936 strahlend schöne, von der Politik ungestörte Olympische Spiele erhofften. In der Zwischenzeit versuchten beide Seiten, sich der Wählerschaft vorteilhaft darzustellen. Dabei zielten die Sozialpolitiker der SPD- wie auch der CDU/CSU-Fraktion insbesondere auf die Rentner.

40 Daten: Peter Schindler, a.a.O. (Fn. 3), Bd. 1, S. 1238 - 1246; Eckart Busch, Die Parlamentsaufösung 1972. Verfassungsgeschichtliche und verfassungsrechtliche Würdigung, in: ZParl, 4. Jg. (1973), H. 2, S. $213-246$, S. 213 f. 


\subsection{Konsequenzen für die gesetzliche Rentenversicherung}

Die dynamische, bruttolohnbezogene Altersrente hatte seit ihrer Einführung 1957 zu einer Anpassung der Renten an vorangegangene Lohnerhöhungen geführt ${ }^{41}$. Aufgrund der Boomphase der deutschen Wirtschaft nach der Rezession von 1966/67 hinkten die Renten den hochgeschnellten Arbeitseinkommen etwas hinterher. Unter der Annahme künftig gleichbleibend hoher Wachstumsraten ließ sich hypothetisch für die nächsten anderthalb Jahrzehnte eine zunehmende „Rentenlücke“ errechnen. Zugleich sahen die Sozialpolitiker von Regierungskoalition und Opposition im Vorgriff auf erwartete Einnahmen einvernehmlich Möglichkeiten zur Ausweitung der Leistungen der Rentenversicherung durch die Einführung einer Mindestrente, die Flexibilisierung der Altersgrenze und die Eröffnung der Möglichkeit zu freiwilliger Nachversicherung für Hausfrauen und Selbständige.

Auf Betreiben der Opposition wurde im September - letztlich einvernehmlich - weiterhin beschlossen, die nächste, routinemäßig für den 1. Januar 1973 anstehende Rentenerhöhung vorzuziehen und rückwirkend zum 1. Juli 1972 in Kraft treten zu lassen. Darüber hinaus setzte die Opposition in populistischer Manier mit ihrer parlamentarischen Mehrheit gegen die Regierungsfraktionen durch, den Beziehern zeitlich vorgezogener Renten unbegrenzte Zuverdienstmöglichkeiten einzuräumen ${ }^{42}$. Die unaufgelöste Konkurrenzsituation hatte somit einen „Sozialwettlauf“ der Konkurrenten - teils zu Gunsten, teils zu Lasten der Versicherten - zur Folge.

Der letztgenannte, sozialpolitisch unsinnige Beschluss, demgemäß es möglich sein sollte, am Ende des Berufslebens doppelte Einkünfte zu beziehen, wurde zu Beginn der 7. Wahlperiode von der erneuerten sozialliberalen Mehrheit unverzüglich zurückgenommen ${ }^{43}$. Aber der Termin für Rentenerhöhungen hatte bei den nachfolgenden Anhebungsbeschlüssen Bestand, so dass sich die 1972 vorgenommene Vorverlegung der Rentenerhöhung als dauerhafte finanzielle Belastung der Versicherungsträger auswirkte (vgl. Tabelle 7).

Im Ergebnis hielten bei der Arbeiterrentenversicherung die Einnahmenzuwächse mit der Zunahme der Ausgaben nicht mehr Schritt. Bei der Knappschaftlichen Rentenversicherung vergrößerte sich das ohnehin vorhandene strukturelle Defizit. Lediglich die Rentenversicherung der Angestellten hatte eine Verminderung ihrer Überschüsse hinzunehmen. Vier Jahre später waren die Belastungen angesichts der tatsächlichen wirtschaftlichen Entwicklung nur noch mit massiven Einschnitten aufzufangen, die das Ausscheiden des schon 1972 verantwortlichen Ministers - Walter Arendt - aus dem Kabinett zur Folge hatten.

41 Zur sozialpolitischen Gesetzgebung Heinz Lampert / Jörg Althammer, Lehrbuch der Sozialpolitik, Berlin u.a. 2007, S. 112, Zeittafel S. 129 - 132.

42 Rentenbeschlüsse: BT-PIPr 6/197 vom 20. September 1972, Abstimmungen S. 11605, S. 11627 , S. 11636, S. 11646; PIPr 6/198 vom 21. September 1972, Schlussabstimmung S. 11711. Zusammenfassende Darstellungen der Ergebnisse bei Manfred G. Schmidt, Sozialpolitik, in: Klaus von Beyme / Manfred G. Schmidt (Hrsg.), Politik in der Bundesrepublik Deutschland, Opladen 1990, S. $126-149$, S. 135.

43 Gesetzentwurf der SPD- und der FDP-Fraktion BT-Drs. 7/3 vom 13. Dezember 1972; Rücknahmebeschluss durch die Verabschiedung des 4. Rentenversicherungs-Änderungsgesetzes, BT-PIPr 7/6 vom 20. Dezember 1972, S. 100. 


\begin{tabular}{|c|c|c|c|c|c|c|c|c|c|}
\hline \multicolumn{10}{|c|}{ Tabelle 7: Defizite und Überschüsse im Bundesetat, 1950 bis 1988 (in Millionen DM) } \\
\hline 1950 & 1951 & 1952 & 1953 & 1954 & 1955 & $\mathbf{1 9 5 6}$ & $\mathbf{1 9 5 7}$ & $\mathbf{1 9 5 8}$ & $\mathbf{1 9 5 9}$ \\
-894 & -1.429 & -203 & +1.641 & +2.783 & +3.615 & +415 & -2.747 & -2.247 & -1.950 \\
\hline $\mathbf{1 9 6 0}$ & $\mathbf{1 9 6 1}$ & $\mathbf{1 9 6 2}$ & $\mathbf{1 9 6 3}$ & $\mathbf{1 9 6 4}$ & $\mathbf{1 9 6 5}$ & $\mathbf{1 9 6 6}$ & $\mathbf{1 9 6 7}$ & $\mathbf{1 9 6 8}$ & $\mathbf{1 9 6 9}$ \\
-60 & +835 & -603 & -2.217 & -266 & -998 & -1.188 & -7.616 & -4.916 & +1.185 \\
\hline $\mathbf{1 9 7 0}$ & $\mathbf{1 9 7 1}$ & $\mathbf{1 9 7 2}$ & $\mathbf{1 9 7 3}$ & $\mathbf{1 9 7 4}$ & $\mathbf{1 9 7 5}$ & $\mathbf{1 9 7 6}$ & $\mathbf{1 9 7 7}$ & $\mathbf{1 9 7 8}$ & $\mathbf{1 9 7 9}$ \\
+353 & -1.385 & -4.777 & -3.199 & -10.337 & -33.083 & -25.856 & -22.206 & -26.313 & -26.053 \\
\hline $\mathbf{1 9 8 0}$ & $\mathbf{1 9 8 1}$ & $\mathbf{1 9 8 2}$ & $\mathbf{1 9 8 3}$ & $\mathbf{1 9 8 4}$ & $\mathbf{1 9 8 5}$ & $\mathbf{1 9 8 6}$ & $\mathbf{1 9 8 7}$ & $\mathbf{1 9 8 8}$ & \\
-27.594 & -37.940 & -37.658 & -31.917 & -28.624 & -22.740 & -23.254 & -27.888 & -39.694 & \\
\hline
\end{tabular}

Quelle: Bundesministerium der Finanzen (Hrsg.), Finanzbericht 1989, Bonn 5. August 1988, S. 157 f.

\subsection{Konsequenzen für den Bundeshaushalt}

Die Haushaltsführung beruhte im gesamten Jahr 1972 auf der Ausnahmeregelung des Art. 111 GG zur vorläufigen Weiterführung der Haushaltswirtschaft. Die hierzu im Dezember 1971 aufgestellten Verwaltungsvorschriften wirkten zunächst restriktiv und legten Höchstgrenzen für die Inanspruchnahme der noch nicht bewilligten Gelder fest. Im Juni wurde darüber hinaus vom Bundeskabinett eine Kürzung der Ausgaben beschlossen. Am 6. September wurde hingegen „[z] ur Erleichterung der vorläufigen Haushaltsführung“ die Beschränkung der verfügbaren Haushaltsansätze aufgehoben. Selbst Ausgaben, die für 1972 erstmals vorgesehen waren und über die noch keinerlei Beschluss des Bundestages gefasst worden war, sollten nun getätigt werden dürfen, wenn der Bundeswirtschafts- und Finanzminister - seit dem 7. Juli 1972 Helmut Schmidt ${ }^{44}$ - sie nach Art. 112 GG genehmigte. Der Große Senat des Bundesrechnungshofes wies zwar auf die strengen Prüfungsmaßstäbe hin, die es hierfür einzuhalten gälte, erhob aber keine grundsätzlichen Einwendungen gegen Ausnahmegenehmigungen im Falle einer ohnehin schon auf einer Ausnahmebestimmung beruhenden Haushaltsführung ${ }^{45}$.

In der Staatsrechtslehre billigen im Anschluss an eine Entscheidung des Bundesverfassungsgerichts zahlreiche Stimmen - ungeachtet der Frage der Korrektheit des Handelns Willy Brandts - die parallele Anwendbarkeit beider Artikel ${ }^{46}$. Eine wachsende Minderheit weist hingegen - zutreffend - auf die nach dem Wortlaut nicht mögliche unmittelbare Anwendung des Artikels 112 in Zeiten provisorischer Haushaltsführung hin, da Art. 112 GG

44 Vgl. Peter Schindler, a.a.O. (Fn. 3), Bd. 1, S. 1078.

45 Vgl. Bundesministerium der Finanzen (Hrsg.), a.a.O. (Fn. 32), S. 92.

46 Vgl. BVerfGE, Bd. 45 Nr. 1 vom 25. Mai 1977, S. 1 - 63, S. 37 (2 BvE 1/74). Hierzu verschiedene Grundgesetzkommentare mit weiteren Nachweisen; für großzügige Verschuldungsmöglichkeiten des Staates im August 1972: Paul Feuchte, Der Nothaushalt - ein Instrument der Politik?, in: AöR, Bd. 97 (1972), S. 538 - 567. Die Auseinandersetzungen fanden in der 7. Wahlperiode des Deutschen Bundestages eine Fortsetzung. Das parlamentsunfreundliche Handeln der Regierung rechtfertigend der SPD-Bundestagsabgeordnete Claus Arndt, Das Verhältnis von Budgetrecht des Parlaments und Zustimmungsrecht des Finanzministers nach Art. 112 GG, in: DVBl. 90. Jg. (1975), S. 601 - 604; mit Bezug auf das Jahr 1973 Finanzminister und Bundesregierung rügend die Entscheidung des Bundesverfassungsgerichts (Zweiter Senat) vom 25. Mai 1977, BVerfGE, Bd. 45 Nr. 1, S. $1-63$. 
einen Staatshaushalt voraussetzt, so dass ohnehin allenfalls eine analoge Anwendung in Betracht käme, die indes dem Ausnahmecharakter beider Normen nicht gerecht würde ${ }^{47}$.

Inhaltlich rechtfertigte die Bundesregierung ihre laufenden Veränderungen des Haushaltsentwurfes mit sich verändernden Informationen zur konjunkturellen Entwicklung ${ }^{48}$. Nach dieser Argumentation hätte eine konjunkturgerechte fiskalische Steuerungspolitik Vorrang vor dem Budgetrecht des Parlaments - eine wohl eher technokratische als demokratische Auffassung ${ }^{49}$.

Im Ergebnis erhöhten sich die Ausgaben für einzelne Aufgabenbereiche drastisch darunter vor allem die Subventionen für den Kohlebergbau, die damalige Deutsche Bundesbahn, das Wohnungswesen samt Wohngeld, aber auch für den Bildungssektor und WestBerlin. Trotz einer Steigerung der Einnahmen um 11,9 Prozent gegenüber 1971 verdreifachte sich das Finanzierungsdefizit des Bundeshaushalts von 1,385 auf 4,777 Milliarden DM. Nachdem der Haushalt des Bundes 1970 letztmalig einen positiven Saldo ausgewiesen hatte, bewegte er sich von da an deutlich ins Negative, in Krisenzeiten und angesichts der deutschen Wiedervereinigung noch drastisch gesteigert, aber in seiner kontinuierlichen Verschuldungstendenz von keiner Regierung mehr verändert.

\section{Resultate und Konsequenzen für die Beurteilung von Minderheitsregierungen}

Aus der Analyse eines einzelnen Falles lassen sich trivialerweise keine allgemeinen Gesetzmäßigkeiten ableiten, wohl aber Hinweise auf Aspekte, die unter strukturell gleichartigen Bedingungen jeweils zu beachten sind.

Konkret hat sich die Relevanz der verfassungsrechtlichen Ausprägung des Misstrauensvotums in seiner „konstruktiv“ genannten Variante gezeigt: Trotz der Minderheitssituation der Regierungskoalition konnte der Kanzler im Amte verbleiben und sich für eine Fortsetzung seiner Politik entscheiden, denn das Grundgesetz gestattet die Fortführung der Kanz-

47 Hierzu die einschlägigen Grundgesetzkommentare: Parlamentsfreundlich-eng zur Interpretation der Art. 111 und 112 Kyrill-A. Schwarz, Art. 111, in: Christian Starck (Hrsg.), Kommentar zum Grundgesetz, begründet von Hermann von Mangoldt, fortgeführt von Friedrich Klein, München 2010, S. 1539 - 1555 und ders., Art. 112, in: ebenda, S. 1556 - 1573; Christoph Gröpl, Art. 111, in: Rudolf Dolzer / Klaus Vogel / Karin Graßhoff(Hrsg.), Bonner Kommentar zum Grundgesetz, Bd. 10, Heidelberg 2001 (Stand: Dezember 2011) ebenso bereits Christoph Sasse, Haushaltsvollzug ohne Haushalt?, in: JZ, 28. Jg. (1973), S. 189 - 192; für enge Interpretation bei paralleler Anwendbarkeit von Art. 111 und 112: Markus Heintzen, Art. 111, in: Grundgesetz. Kommentar, Bd. 2, begründet von Ingo von Münch, hrsg. von Philip Kunig, München 2012, S. 1257 - 1264; ders., Art. 112, in: ebenda, S. 1265 - 1273; exekutivfreundlich-weit Hanno Kube, Art. 111 (2007), in: Grundgesetz. Kommentar, begründet von Theodor Maunz / Günter Dürig, hrsg. von Roman Herzog / Rupert Scholz / Matthias Herdegen / Hans H. Klein, München 2011; Werner Heun, Artikel 111, in: Horst Dreier (Hrsg.), Grundgesetz. Kommentar, Tübingen 2008, S. 1059 - 1066 (für analoge Anwendbarkeit des Art. 112 unter den Bedingungen des Art. 111 GG); Hans Bernhard Brockmeyer, Art. 111, in: Hans Hofmann / Axel Hopf (Hrsg.), GG. Kommentar zum Grundgesetz, begründet von Bruno Schmidt-Bleibtreu / Franz Klein, Köln 2011, S. 2301 ff. Bei vorangegangener Ablehnung des Etatentwurfs durch das Parlament - wie im vorliegenden Falle - hält Brockmeyer die Anwendung des Art. 112 i.V.m. Art. 111 GG jedoch explizit für verfassungswidrig, Rdnr. 5.

48 Bundesministerium der Finanzen (Hrsg.), a.a.O. (Fn. 32), S. 93.

49 Hinsichtlich der konjunkturpolitischen Argumentation ebenso Markus Heintzen, a.a.O. (Fn. 47), Art. 111, Rdnr. 5. 
lerschaft bis zur Wahl eines Nachfolgers. Da Brandt bereits bei der Abstimmung über den gegen ihn gerichteten Misstrauensantrag in eine Minderheitsposition geraten war, hat er sein Amt von diesem Zeitpunkt an allein aufgrund der verfassungsrechtlichen Regelung ausgeübt.

Das bedeutet: Willy Brandt hat vom 27. April bis zu seiner Wiederwahl zum Bundeskanzler am 14. Dezember 1972 siebeneinhalb Monate ohne parlamentarische Mehrheit, davon fünfeinhalb Monate ohne ein handlungsfähiges Parlament ${ }^{50}$ und das ganze Jahr 1972 hindurch ohne Staatshaushalt regiert. Dabei sind die haushaltspolitischen Probleme seiner Regierung erst nach dem nicht zum Regierungswechsel führenden Misstrauensvotum eingetreten. Im Gegensatz zu der von Markus Heintzen gegebenen Empfehlung, einen Haushaltskonflikt mittels Art. 67 GG zu beenden ${ }^{51}$, hat diese Vorschrift historisch-konkret gerade den Anfang der Konfliktphase markiert.

Haushaltsrechtlich ist die Amtsführung der Bundesregierung im Jahre 1972 ausdrücklich auf die Regelung des Art. 111 Abs. 1 GG aus der vordemokratischen Regierungspraxis Otto von Bismarcks gestützt worden. Ebenso wie Bismarck ist Brandt dafür nach erfolgreich durchstandener Krise elektoral belohnt und parlamentarisch absolutiert worden. Insoweit hat sich die Phase haushaltslosen Regierens unter Aspekten der Machterhaltung für beide Politiker gelohnt.

Differenzierter fällt die Bilanz aus, wenn man die während der Phase der Minderheitsregierung beschlossenen politischen Inhalte betrachtet: Nur in zwei Fällen gelang der Regierungsseite noch eine Verabschiedung von Gesetzen gegen die Opposition. Bei insgesamt 57 außenwirksamen Schlussabstimmungen zu Gesetzen (einschließlich der Vertragsgesetze) ohne die fünf, sämtlich einmütig beschlossenen parlamentarischen Entschließungen - ergibt sich daraus eine Quote von 96,5 Prozent einvernehmlich oder ohne oppositionelle Herausforderung der Regierungskoalition verabschiedeter zu 3,5 Prozent seitens der Regierungskoalition allein bestimmter Endergebnisse.

Mangels eigener Mehrheit, die bereits im Falle eines Mandatsgleichstands mit der Opposition fehlt, sind Minderheitsregierungen für inhaltliche Erfolge parlamentarisch strukturell jeweils auf das Einvernehmen mit mehr oder weniger großen Teilen der nicht-gouvernementalen Fraktionen angewiesen. Im konkreten Falle hat dies auch für die in ,antagonistischer Kooperation" 52 zum Abschluss gebrachten Ostverträge gegolten. Aus der legislatorischen Bilanz nach dem Mehrheitsverlust ist danach eine inhaltliche Rechtfertigung für den Verbleib im Amte im Sinne einer politisch-programmatischen „Notwendigkeit“ kaum abzuleiten.

Auf der Seite der Opposition sind die Bedingungen für politisch-rationales Handeln zu Zeiten einer Minderheitsregierung im Vergleich zu Phasen mit parlamentarisch mehrheitsfähigen Regierungsfraktionen gleichfalls verändert: Da oppositionelle Initiativen eventuell auch ohne Zustimmung der Regierungsfraktionen verabschiedet werden können, bestehen mehrere strategische Optionen:

50 Die Zeitangabe errechnet sich aus der dreimonatigen parlamentarischen Sommerpause vom 23. Juni bis zum 20. September 1972 und dem gemäß Art. 39 und 45 a.F. GG mit der Auflösung des 6. Bundestages am 22. September 1972 bis zur Konstituierung des 7. am 13. Dezember 1972 eingetretenen parlamentslosen Zustand.

51 Vgl. Markus Heintzen, a.a.O. (Fn. 47), Art. 112, Rdnr. 4.

52 Werner Link, Die CDU/CSU-Fraktion und die neue Ostpolitik, a.a.O. (Fn. 21), S. 138. 
- eine harte Verweigerungshaltung, um die Regierung durch parlamentarische Niederlagen öffentlich zu demütigen und zur freiwilligen Demission zu bewegen,

- eine kooperative Haltung, um die regierende Minderheit zu inhaltlichen Zugeständnissen nach Vorstellungen der Opposition zu veranlassen und

- die Lancierung eigener Initiativen, die eine Chance haben, eine parlamentarische Mehrheit zu finden und dadurch Gestaltungswillen und Gestaltungsfähigkeit der Opposition dokumentieren können.

Anders als von Carlo Schmid in seiner klassischen Beschreibung gesamtstaatlich wesentlicher Funktionen der Opposition betont ${ }^{53}$, reduziert sich demgegenüber der potentielle politische Nutzen des Verweises auf Prinzipien, die möglicherweise von der Wählerschaft akzeptiert werden, in der praktischen Regierungsarbeit hingegen verletzt worden sein können. Dies gilt etwa für die Anmahnung eines sparsamen Umgangs mit Haushaltsmitteln - insbesondere dann, wenn gesamtgesellschaftlich eine andere Werthaltung weit verbreitet ist.

Konkret ist in der laufenden Wahlperiode nach dem Scheitern des Misstrauensantrages keine weitere Initiative zur Ablösung der Bundesregierung mehr erfolgt. Überwiegend lässt sich vielmehr seitens der Opposition die Verfolgung der zweiten Alternative beobachten, die auch ihrem vorangegangenen Abstimmungsverhalten entsprochen hat.

Demgegenüber hat die Opposition im Sinne der dritten Alternative in der China- und Rentenpolitik die Minderheitsregierung in die Position eines getriebenen Akteurs zu versetzen versucht. Die dabei erzielten inhaltlichen Erfolge haben sich indes elektoral für die Opposition nicht ausgezahlt.

In der Gesamtbilanz ergibt sich aus diesen Beobachtungen: Die unter den gegebenen parlamentarischen Mehrheitsverhältnissen nicht ablösbare Minderheitsregierung hat sich hinsichtlich der Ausübung ihrer politischen Führungsfunktion in einer labilen Position befunden. Denn eine lediglich „tolerierte“ Minderheitsregierung ist strukturell nicht in der Lage, konkurrierende Initiativen der Opposition aus eigener Kraft einfach ,auszusitzen“ oder „abzuwettern“. Hierdurch ist im konkreten Fall der Rentenversicherung die von jeder Regierung situativ anzustrebende Mischung aus begünstigenden und belastenden Beschlüssen in Richtung einer Ausgabensteigerung verschoben worden. Die parlamentarisch nicht restringierte Anwendung problematischer Bestimmungen des Haushaltsrechts hat parallel dazu den Bundeshaushalt deutlich über die Höhe der laufenden Einnahmen hinaus anwachsen lassen.

Die „offene Flanke“ der Bundesregierung im Parlament hat ihr machtpolitisch letztlich nicht geschadet, die Opposition hingegen politisch-strategisch vor besondere Herausforderungen gestellt, die sie nicht zur eigenen Zufriedenheit hat bewältigen können. Durch den erkennbar gewordenen Wettkampf um die populärere Sozialpolitik hat sich die parlamentarische Schwäche der Regierungskoalition indes belastend auf die öffentlichen Finanzen ausgewirkt - ein Aspekt, der zur Abschätzung der Attraktivität von Minderheitsregierungen auch künftig nicht übersehen werden sollte.

53 Vgl. Carlo Schmid, Die Opposition als Staatseinrichtung (1955), abgedruckt in: Hans-Gerd Schumann (Hrsg.), Die Rolle der Opposition in der Bundesrepublik Deutschland, Darmstadt 1976, S. 53 - 65, vor allem S. $59-63$. 